\title{
Fixed and common fixed point theorems in partially ordered quasi-metric spaces
}

\author{
Wasfi Shatanawi ${ }^{a, b}$, Mohd Salmi MD Nooranic ${ }^{c}$ Habes Alsamir ${ }^{c, *}$, Anwar Bataihah ${ }^{d}$ \\ ${ }^{a}$ Department of Mathematics and general courses, Prince Sultan University, Riyadh, Saudi Arabia. \\ ${ }^{b}$ Department of Mathematics, Hashemite University, Zarqa, Jordan. \\ 'School of Mathematical Sciences, Faculty of Science and Technology, Universiti Kebangsaan, Malaysia, 43600 UKM, \\ Selangor. \\ ${ }^{d}$ Department of Mathematics, Faculty of Science, Irbid National University, Irbid, Jordan.
}

\begin{abstract}
In this paper, we prove some new fixed and common fixed point results in the framework of partially ordered quasi-metric spaces under linear and nonlinear contractions. Also we obtain some fixed point results in the framework of G-metric spaces. (C)2016 All rights reserved.

Keywords: Quasi metric, common fixed point theorem, nonlinear contraction, altering distance function, G-metric spaces.

2010 MSC: 54H25, 47H10.
\end{abstract}

\section{Introduction and preliminaries}

The fixed point theory is considered as a basic and very simple mathematical setting, since it has some applications in many interesting fields such as differential equations, economics and engineering. The existence of a fixed point is a pivotal property of a function. Many necessary or sufficient conditions for the presence of such points are considered in many areas in mathematics.

The Banach contraction theorem [4] is considered as a fundamental theorem concerning fixed point theorem in a complete metric space which is appeared in 1922 and rise for its elegant and simple proof which it is known later as Banach contraction principle. Subsequently, a large number

*Corresponding author

Email addresses: wshatanawi@psu.edu.sa (Wasfi Shatanawi), msn@ukm.my (Mohd Salmi MD Noorani), h.alsamer@gmail.com (Habes Alsamir), anwerbataihah@gmail.com (Anwar Bataihah) 
of generalizations of Banach contraction principle were obtained, for example in 2008, Agarwal et al. [1] introduced and proved the following theorem.

Theorem 1.1 ([1, Theorem 2.3]). Let $(X, d, \preceq)$ be a partially ordered complete metric space. Assume $\psi:[0,+\infty) \rightarrow[0,+\infty)$ is a non-decreasing function with $\psi(t)<t$ for each $t>0$. Moreover, suppose that $f$ is a nondecreasing mapping satisfying the following form

$$
d(f(x), f(y)) \leq \psi(\max \{f d(x, y), d(x, f(x)), d(y, f(y))\})
$$

for all $x \geq y$. Also assume either $f$ is continuous or if $\left(x_{n}\right) \subseteq X$ is a nondecreasing sequence with $x_{n}$ $\rightarrow x$ in $X$, then $x_{n} \leq x$ for all $n$ holds. If there exists an $x_{0} \in X$ with $x_{0} \leq f\left(x_{0}\right)$ then $f$ has a fixed point.

The concept of quasi-metric spaces was generated by Wilson [19] in 1931 as the following:

Definition 1.2. Let $X$ be a non empty set and $d: X \times X \rightarrow[0, \infty)$ be a given function which satisfies

(1) $d(x, y)=0$ iff $x=y$;

(2) $d(x, y) \leq d(x, z)+d(z, y)$ for any points $x, y, z \in X$.

Then $d$ is called a quasi metric on $X$ and the pair $(X, d)$ is called a quasi metric space.

It is clear that every metric space is a quasi metric space, but the reverse is not necessarily true.

Jleli and Samet [5] and Samet et al. [16] utilized the notion of quasi-metric space to obtain some fixed point theorems. In their interesting papers, they pointed out that some fixed point results in $G$-metric space in sense of Mustafa and Sims [14] can be obtained from quasi-metric space. Agarwal et. al [2] showed that many fixed point theorems in $G$-metric spaces can be derived from known existing results if all arguments are not distinct. For some results in $G$-metric space, we refer the reader to [7-18].

The convergence and completeness in a quasi-metric space are defined as follows:

Definition $1.3([5])$. Let $(X, d)$ be a quasi-metric space, $\left(x_{n}\right)$ be a sequence in $X$, and $x \in X$. Then the sequence $\left(x_{n}\right)$ converges to $x$ if and only if $\lim _{n \rightarrow \infty} d\left(x_{n}, x\right)=\lim _{n \rightarrow \infty} d\left(x, x_{n}\right)=0$.

Definition $1.4([5])$. Let $(X, d)$ be a quasi-metric space and $\left(x_{n}\right)$ be a sequence in $X$. We say that the sequence $\left(x_{n}\right)$ is left-Cauchy if for every $\epsilon>0$ there is positive integer $N=N(\epsilon)$ such that $d\left(x_{n}, x_{m}\right) \leq \epsilon$ for all $n \geq m>N$.

Definition $1.5([5])$. Let $(X, d)$ be a quasi-metric space and $\left(x_{n}\right)$ be a sequence in $X$. We say that the sequence $\left(x_{n}\right)$ is right-Cauchy if for every $\epsilon>0$ there is a positive integer $N=N(\epsilon)$ such that $d\left(x_{n}, x_{m}\right) \leq \epsilon$ for all $m \geq n>N$.

Definition $1.6([5])$. Let $(X, d)$ be a quasi-metric space and $\left(x_{n}\right)$ be a sequence in $X$. We say that the sequence $\left(x_{n}\right)$ is Cauchy if for every $\epsilon>0$ there is positive integer $N=N(\epsilon)$ such that $d\left(x_{n}, x_{m}\right) \leq \epsilon$ for all $m, n>N$.

Definition $1.7([5])$. Let $(X, d)$ be a quasi-metric space. We say that

(1) $(X, d)$ is left-complete if and only if every left-Cauchy sequence in $X$ is convergent;

(2) $(X, d)$ is right-complete if and only if every right-Cauchy sequence in $X$ is convergent;

(3) $(X, d)$ is complete if and only if every Cauchy sequence in $X$ is convergent. 
Mustafa and Sims [14] introduced the notion of $G$-metric spaces as follows:

Definition $1.8([14])$. Let $X$ be a nonempty set and let $G: X \times X \times X \rightarrow[0, \infty)$ be a function satisfying:

(G1) $G(x, y, z)=0$ if $x=y=z$,

(G2) $G(x, x, y)>0$ for all $x, y \in X$, with $x \neq y$,

(G3) $G(x, y, y) \leq G(x, y, z)$ for all $x, y, z \in X$, with $y \neq z$,

(G4) $G(x, y, z)=G(p\{x, y, z\})$, where $p\{x, y, z\}$ is the all possible permutations of $\{x, y, z\}$ (symmetry),

(G5) $G(x, y, z) \leq G(x, a, a)+G(a, y, z) \forall x, y, z, a \in X$ (rectangle inequality).

Then the function $\mathrm{G}$ is called a generalized metric, or more specifically a G-metric on $X$, and the pair $(X, G)$ is called a $\mathrm{G}$-metric space.

Definition $1.9([14])$. Let $(X, G)$ be a G-metric space and let $\left(x_{n}\right)$ be a sequence of points of $X$. Then we say that $\left(x_{n}\right)$ is G-convergent to $x$ if $\lim _{n, m \rightarrow \infty} G\left(x, x_{n}, x_{m}\right)=0$; that is, for any $\epsilon>0$, there exists $k \in \mathbb{N}$ such that $G\left(x, x_{n}, x_{m}\right)<\epsilon$, for all $n, m \geq k$.

Proposition 1.10 ([14]). Let $(X, G)$ be a G-metric space. Then the following assertions are equivalent

(1) $\left(x_{n}\right)$ is G-convergent to $x$;

(2) $G\left(x_{n}, x_{n}, x\right) \rightarrow 0$ as $n \rightarrow \infty$;

(3) $G\left(x_{n}, x, x\right) \rightarrow 0$ as $n \rightarrow \infty$.

Definition $1.11([14])$. Let $(X, G)$ be a G-metric space. A sequence $\left(x_{n}\right)$ in $X$ is said to be G-Cauchy if for every $\epsilon>0$, there exists $k \in \mathbb{N}$ such that

$$
G\left(x_{n}, x_{m}, x_{l}\right)<\epsilon, \quad \forall n, m, l \geq k .
$$

Proposition 1.12 ([14]). In a G-metric space, the following are equivalent

(1) the sequence $\left(x_{n}\right)$ is G-Cauchy;

(2) for every $\epsilon>0$, there exists $k \in \mathbb{N}$ such that $G\left(x_{n}, x_{m}, x_{m}\right)<\epsilon$ for all $n, m \geq k$.

Definition 1.13 ([14]). A G-metric space $(X, G)$ is said to be G-complete or complete G-metric space if every G-Cauchy sequence in $(X, G)$ is $\mathrm{G}$-convergent in $(X, G)$.

The following theorem is a relation between G-metric spaces and quasi metric spaces.

Theorem $1.14([5])$. Let $(X, G)$ be a G-metric space and let $d: X \times X \rightarrow[0, \infty)$ defined by $d(x, y)=G(x, y, y)$. Then

(1) $(X, d)$ is a quasi metric space;

(2) $\left(x_{n}\right) \subset X$ is $G$-convergent to $x \in X$ iff $\left(x_{n}\right)$ is convergent in $(X, d)$;

(3) $\left(x_{n}\right) \subset X$ is G-Cauchy iff $\left(x_{n}\right)$ is Cauchy in $(X, d)$;

(4) $\left(x_{n}\right) \subset X$ is $G$-complete iff $\left(x_{n}\right)$ is complete in $(X, d)$. 


\section{Main result}

We start with the following definitions.

Definition $2.1([3])$. Let $(X, \preceq)$ be a partially ordered set. Two mappings $F, G: X \rightarrow X$ are said to be weakly increasing if $F x \preceq G F x$ and $G x \preceq F G x$, for all $x \in X$.

Definition $2.2([18])$. Let $(X, \preceq)$ be a partially ordered set and $A, B$ be closed subsets of $X$ with $X=A \cup B$. Let $f, g: X \rightarrow X$ be two mappings. Then the pair $(f, g)$ is said to be $(A, B)$-weakly increasing if $f x \preceq g f x$ for all $x \in A$ and $g x \preceq f g x$ for all $x \in B$.

Definition $2.3([6])$. The function $\phi:[0, \infty) \rightarrow[0, \infty)$ is called an altering distance function if the following properties are satisfied.

(1) $\phi$ is continuous and nondecreasing.

(2) $\phi(t)=0$ if and only if $t=0$.

Our main result in this section is the following theorem.

Theorem 2.4. Let $(X, \preceq)$ be a partially ordered set and suppose that $(X, d)$ is a complete quasimetric space. Let $A, B$ be two nonempty closed subsets of $X$ with respect to the topology induced by $d$ with $X=A \cup B$ and $A \cap B \neq \phi$. Let $f, g: A \cup B \rightarrow A \cup B$ such that the pair $(f, g)$ is (A,B)-weakly increasing with $f(A) \subseteq B, g(B) \subseteq A$. Let $\phi, \psi$ be altering distance functions. Moreover, suppose that

$$
\phi d(f x, g y) \leq \phi \max \{d(x, y), d(f x, x), d(g y, y)\}-\psi \max \{d(x, y), d(f x, x), d(g y, y)\}
$$

for all comparative $x, y \in X$ with $x \in A, y \in B$, and

$$
\phi d(g x, f y) \leq \phi \max \{d(x, y), d(g x, x), d(f y, y)\}-\psi \max \{d(x, y), d(g x, x), d(f y, y)\}
$$

for all comparative $x, y \in X$ with $x \in B, y \in A$. Also,

1. suppose that there exists $x_{0} \in A$ such that $x_{0} \preceq f x_{0}$,

2. if $f$ or $g$ is continuous.

Then $f$ and $g$ have a common fixed point in $A \cap B$.

Proof. From 1. there exists $x_{0} \in A$ such that $x_{0} \preceq f x_{0}$. Since $f(A) \subseteq B$, then $x_{1}=f x_{0} \in B$. Also, since $g(B) \subseteq A$, then $x_{2}=g x_{1} \in B$. By continuing this way, we construct a sequence $\left(x_{n}\right)$ in $X$ such that $f x_{2 n}=x_{2 n+1}, x_{2 n} \in A, g x_{2 n+1}=x_{2 n+2}$ and $x_{2 n+1} \in B, n \in \mathbb{N} \cup\{0\}$. Since $(f, g)$ is $(A, B)$-weakly increasing, then $x_{0} \preceq f x_{0}=x_{1} \preceq g f x_{0}=g x_{1}=x_{2} \preceq f g x_{1}=f x_{2}=x_{3} \cdots$. Thus $x_{n} \preceq x_{n+1}$ for all $n \geq 0$. If there exists some $k \in \mathbb{N}$ such that $x_{2 k}=x_{2 k+1}$, then $x_{2 k}$ is a fixed point for $f$ in $A \cap B$. To show that $x_{2 k}$ is also a fixed point for $g$ it is equivalent to show that $x_{2 k}=x_{2 k+1}=x_{2 k+2}$. Since $x_{2 k} \preceq x_{2 k+1}$, then by $(2.2)$ we have

$$
\begin{aligned}
\phi d\left(x_{2 k+2}, x_{2 k+1}\right)= & \phi d\left(g x_{2 k+1}, f x_{2 k}\right) \\
\leq & \phi \max \left\{d\left(x_{2 k+1}, x_{2 k}\right), d\left(x_{2 k+2}, x_{2 k+1}\right), d\left(x_{2 k+1}, x_{2 k}\right)\right\} \\
& -\psi \max \left\{d\left(x_{2 k+1}, x_{2 k}\right), d\left(x_{2 k+2}, x_{2 k+1}\right), d\left(x_{2 k+1}, x_{2 k}\right)\right\} \\
\leq & \phi d\left(x_{2 k+2}, x_{2 k+1}\right)-\psi d\left(x_{2 k+2}, x_{2 k+1}\right) .
\end{aligned}
$$

Therefore, $\psi d\left(x_{2 k+2}, x_{2 k+1}\right)=0$, and so $d\left(x_{2 k+2}, x_{2 k+1}\right)=0$. Hence $x_{2 k+2}=x_{2 k+1}$. Thus $x_{2 k}$ is a common fixed point for $f$ and $g$ in $A \cap B$. 
Now, assume that $x_{n} \neq x_{n+1}$ for all $n \geq 0$. Let $n \in \mathbb{N}$. If $n$ is even, then $n=2 t$ for some $t \in \mathbb{N}$. By (2.1), we have

$$
\begin{aligned}
\phi d\left(x_{n+1}, x_{n}\right)=\phi d\left(x_{2 t+1}, x_{2 t}\right)= & \phi d\left(f x_{2 t}, g x_{2 t-1}\right) \\
\leq & \phi \max \left\{d\left(x_{2 t}, x_{2 t-1}\right), d\left(x_{2 t+1}, x_{2 t}\right), d\left(x_{2 t}, x_{2 t-1}\right)\right\} \\
& -\psi \max \left\{d\left(x_{2 t}, x_{2 t-1}\right), d\left(x_{2 t+1}, x_{2 t}\right), d\left(x_{2 t}, x_{2 t-1}\right)\right\} \\
\leq & \phi \max \left\{d\left(x_{2 t+1}, x_{2 t}\right), d\left(x_{2 t}, x_{2 t-1}\right)\right\} \\
& -\psi \max \left\{d\left(x_{2 t+1}, x_{2 t}\right), d\left(x_{2 t}, x_{2 t-1}\right)\right\} .
\end{aligned}
$$

If $\max \left\{d\left(x_{2 t+1}, x_{2 t}\right), d\left(x_{2 t}, x_{2 t-1}\right)\right\}=d\left(x_{2 t+1}, x_{2 t}\right)$, then

$$
\phi d\left(x_{2 t+1}, x_{2 t}\right) \leq \phi d\left(x_{2 t+1}, x_{2 t}\right)-\psi d\left(x_{2 t+1}, x_{2 t}\right) .
$$

Therefore, $\psi d\left(x_{2 t+1}, x_{2 t}\right)=0$ and so $d\left(x_{2 t+1}, x_{2 t}\right)=0$. Thus $x_{2 t+1}=x_{2 t}$ is a contradiction. Hence $\max \left\{d\left(x_{2 t+1}, x_{2 t}\right), d\left(x_{2 t}, x_{2 t-1}\right)\right\}=d\left(x_{2 t}, x_{2 t-1}\right)$. Therefore

$$
d\left(x_{n+1}, x_{n}\right) \leq d\left(x_{n}, x_{n-1}\right)
$$

and

$$
\phi d\left(x_{n+1}, x_{n}\right) \leq \phi d\left(x_{n}, x_{n-1}\right)-\psi d\left(x_{n}, x_{n-1}\right) .
$$

If $n$ is odd, then $n=2 t+1$ for some $t \in \mathbb{N}$. By (2.2), we have

$$
\begin{aligned}
\phi d\left(x_{n+1}, x_{n}\right)=\phi d\left(x_{2 t+2}, x_{2 t+1}\right)= & \phi d\left(g x_{2 t+1}, f x_{2 t}\right) \\
\leq & \phi \max \left\{d\left(x_{2 t+1}, x_{2 t}\right), d\left(x_{2 t+2}, x_{2 t+1}\right), d\left(x_{2 t+1}, x_{2 t}\right)\right\} \\
& -\psi \max \left\{d\left(x_{2 t+1}, x_{2 t}\right), d\left(x_{2 t+2}, x_{2 t+1}\right), d\left(x_{2 t+1}, x_{2 t}\right)\right\} \\
\leq & \phi \max \left\{d\left(x_{2 t+1}, x_{2 t}\right), d\left(x_{2 t+2}, x_{2 t+1}\right)\right\} \\
& -\psi \max \left\{d\left(x_{2 t+1}, x_{2 t}\right), d\left(x_{2 t+2}, x_{2 t+1}\right)\right\} .
\end{aligned}
$$

If $\max \left\{d\left(x_{2 t+1}, x_{2 t}\right), d\left(x_{2 t+2}, x_{2 t+1}\right)\right\}=d\left(x_{2 t+2}, x_{2 t+1}\right)$, then $\phi d\left(x_{2 t+2}, x_{2 t+1}\right) \leq \phi d\left(x_{2 t+2}, x_{2 t+1}\right)-$ $\psi d\left(x_{2 t+2}, x_{2 t+1}\right)$. Therefore, $\psi d\left(x_{2 t+2}, x_{2 t+1}\right)=0$, and so $d\left(x_{2 t+2}, x_{2 t+1}\right)=0$. Thus $x_{2 t+2}=x_{2 t+1}$ is a contradiction. Hence $\max \left\{d\left(x_{2 t+1}, x_{2 t}\right), d\left(x_{2 t+2}, x_{2 t+1}\right)\right\}=d\left(x_{2 t+1}, x_{2 t}\right)$. Therefore,

$$
d\left(x_{n+1}, x_{n}\right) \leq d\left(x_{n}, x_{n-1}\right)
$$

and

$$
\phi d\left(x_{n+1}, x_{n}\right) \leq \phi d\left(x_{n}, x_{n-1}\right)-\psi d\left(x_{n}, x_{n-1}\right) .
$$

From (2.3) and (2.5), we have for all $n \in \mathbb{N}$

$$
d\left(x_{n+1}, x_{n}\right) \leq d\left(x_{n}, x_{n-1}\right) .
$$

Thus $\left(d\left(x_{n+1}, x_{n}\right): n \in \mathbb{N}\right)$ is a nonnegative decreasing sequence, so there exists $r \geq 0$ such that $\lim _{n \rightarrow \infty} d\left(x_{n+1}, x_{n}\right)=r$. Also, from 2.4 and 2.6 , we have for all $n \in \mathbb{N}$

$$
\phi d\left(x_{n+1}, x_{n}\right) \leq \phi d\left(x_{n}, x_{n-1}\right)-\psi d\left(x_{n}, x_{n-1}\right) .
$$

By taking the limit as $n \rightarrow \infty$ in (2.8), we get $\phi r \leq \phi r-\psi r$ which implies that $\psi r=0$. Therefore, $r=0$. Thus

$$
\lim _{n \rightarrow \infty} d\left(x_{n+1}, x_{n}\right)=0 .
$$


Again, let $n \in \mathbb{N}$. If $n$ is even, then $n=2 t$ for some $t \in \mathbb{N}$. By $(2.2)$, we have

$$
\begin{aligned}
\phi d\left(x_{n}, x_{n+1}\right)=\phi d\left(x_{2 t}, x_{2 t+1}\right)= & \phi d\left(g x_{2 t-1}, f x_{2 t}\right) \\
\leq & \phi \max \left\{d\left(x_{2 t-1}, x_{2 t}\right), d\left(x_{2 t}, x_{2 t-1}\right), d\left(x_{2 t+1}, x_{2 t}\right)\right\} \\
& -\psi \max \left\{d\left(x_{2 t-1}, x_{2 t}\right), d\left(x_{2 t}, x_{2 t-1}\right), d\left(x_{2 t+1}, x_{2 t}\right)\right\} .
\end{aligned}
$$

From (2.7), we have $d\left(x_{2 t}, x_{2 t-1}\right)>d\left(x_{2 t+1}, x_{2 t}\right)$. Thus

$$
\begin{aligned}
\phi d\left(x_{2 t}, x_{2 t+1}\right) \leq & \phi \max \left\{d\left(x_{2 t-1}, x_{2 t}\right), d\left(x_{2 t}, x_{2 t-1}\right)\right\} \\
& -\psi \max \left\{d\left(x_{2 t-1}, x_{2 t}\right), d\left(x_{2 t}, x_{2 t-1}\right)\right\} \\
\leq & \phi \max \left\{d\left(x_{2 t-1}, x_{2 t}\right), d\left(x_{2 t}, x_{2 t-1}\right)\right\} .
\end{aligned}
$$

Since $\phi$ is an altering distance function, then

$$
d\left(x_{2 t}, x_{2 t+1}\right) \leq \max \left\{d\left(x_{2 t-1}, x_{2 t}\right), d\left(x_{2 t}, x_{2 t-1}\right)\right\} .
$$

From (2.7) we have

$$
d\left(x_{2 t+1}, x_{2 t}\right) \leq d\left(x_{2 t}, x_{2 t-1}\right) \leq \max \left\{d\left(x_{2 t-1}, x_{2 t}\right), d\left(x_{2 t}, x_{2 t-1}\right)\right\} .
$$

From (2.11) and 2.12), we have

$$
\max \left\{d\left(x_{2 t+1}, x_{2 t}\right), d\left(x_{2 t}, x_{2 t+1}\right)\right\} \leq \max \left\{d\left(x_{2 t-1}, x_{2 t}\right), d\left(x_{2 t}, x_{2 t-1}\right)\right\} .
$$

Similarly, we can show that

$$
\max \left\{d\left(x_{2 t+1}, x_{2 t+2}\right), d\left(x_{2 t+2}, x_{2 t+1}\right)\right\} \leq \max \left\{d\left(x_{2 t+1}, x_{2 t}\right), d\left(x_{2 t}, x_{2 t+1}\right)\right\} .
$$

From 2.13 and 2.14 , we get that

$$
\max \left\{d\left(x_{n}, x_{n+1}\right), d\left(x_{n+1}, x_{n}\right)\right\} \leq \max \left\{d\left(x_{n}, x_{n-1}\right), d\left(x_{n-1}, x_{n}\right)\right\} \text { holds for all } \mathrm{n} \in \mathbb{N} \text {. }
$$

So $\left(\max \left\{d\left(x_{n}, x_{n+1}\right), d\left(x_{n+1}, x_{n}\right)\right\}\right)$ is a nonnegative decreasing sequence. Hence there exists $r \geq 0$ such that

$$
\lim _{n \rightarrow \infty} \max \left\{d\left(x_{n}, x_{n+1}\right), d\left(x_{n+1}, x_{n}\right)\right\}=r .
$$

From (2.9), we get

$$
\lim _{n \rightarrow \infty} d\left(x_{n}, x_{n+1}\right)=r
$$

From 2.10, we get

$$
\phi(r) \leq \phi(r)-\psi(r) .
$$

So $\psi(r)=0$, and hence $r=0$. Therefore, for all $n \in \mathbb{N}$

$$
\lim _{n \rightarrow \infty} d\left(x_{n}, x_{n+1}\right)=0 .
$$

Now, our claim is to show that $\left(x_{n}\right)$ is Cauchy sequence. To show that $\left(x_{n}\right)$ is a Cauchy sequence it is sufficient to show that $\left(x_{2 n}\right)$ is a Cauchy sequence; that is $\left(x_{2 n}\right)$ is left-Cauchy and right-Cauchy. Suppose to the contrary that $\left(x_{2 n}\right)$ is not left-Cauchy. Then there is $\epsilon>0$ and two subsequences $\left(x_{2 n_{k}}\right)$ and $\left(x_{2 m_{k}}\right)$ such that $\left(x_{2 n_{k}}\right)$ chosen to be the smallest index for which

$$
d\left(x_{2 n_{k}}, x_{2 m_{k}}\right) \geq \epsilon \quad 2 n_{k}>2 m_{k}>k .
$$


This means that

$$
d\left(x_{2 n_{k}-2}, x_{2 m_{k}}\right)<\epsilon
$$

From (2.15), we get

$$
\begin{aligned}
\epsilon \leq d\left(x_{2 n_{k}}, x_{2 m_{k}}\right) & \leq d\left(x_{2 n_{k}}, x_{2 n_{k}-1}\right)+d\left(x_{2 n_{k}-1}, x_{2 m_{k}}\right) \\
& \leq d\left(x_{2 n_{k}}, x_{2 n_{k}-1}\right)+d\left(x_{2 n_{k}-1}, x_{2 n_{k}-2}\right)+d\left(x_{2 n_{k}-2}, x_{2 m_{k}}\right) .
\end{aligned}
$$

Taking the limit as $k \rightarrow \infty$ and using (2.9), we conclude

$$
\lim _{k \rightarrow \infty} d\left(x_{2 n_{k}-1}, x_{2 m_{k}}\right)=\epsilon .
$$

Again, from 2.15, we obtain

$$
\epsilon \leq d\left(x_{2 n_{k}}, x_{2 m_{k}}\right) \leq d\left(x_{2 n_{k}}, x_{2 m_{k}+1}\right)+d\left(x_{2 m_{k}+1}, x_{2 m_{k}}\right) .
$$

Taking the limit as $k \rightarrow \infty$ and using (2.9), we see that

$$
\epsilon \leq \lim _{k \rightarrow \infty} d\left(x_{2 n_{k}}, x_{2 m_{k}+1}\right) .
$$

The contraction condition 2.2 yields

$$
\begin{aligned}
\phi d\left(x_{2 n_{k}}, x_{2 m_{k}+1}\right)= & \phi d\left(g x_{2 n_{k}-1}, f x_{2 m_{k}}\right) \\
\leq & \phi \max \left\{d\left(x_{2 n_{k}-1}, x_{2 m_{k}}\right), d\left(x_{2 n_{k}}, x_{2 n_{k}-1}\right), d\left(x_{2 m_{k}+1}, x_{2 m_{k}}\right)\right\} \\
& -\psi \max \left\{d\left(x_{2 n_{k}-1}, x_{2 m_{k}}\right), d\left(x_{2 n_{k}}, x_{2 n_{k}-1}\right), d\left(x_{2 m_{k}+1}, x_{2 m_{k}}\right)\right\} .
\end{aligned}
$$

Taking the limit as $k \rightarrow \infty$ and using the continuity of $\phi, \psi$ and using $(2.9),(2.16)$, and (2.17), we get

$$
\phi \epsilon \leq \phi \lim _{k \rightarrow \infty} d\left(x_{2 n_{k}}, x_{2 m_{k}+1}\right) \leq \phi \epsilon-\psi \epsilon .
$$

Therefore, $\psi \epsilon=0$, and hence $\epsilon=0$ which is 1a contradiction since $\epsilon>0$. Hence $\left(x_{2 n}\right)$ is a left-Cauchy sequence. In a similar manner we can prove that $\left(x_{2 n}\right)$ is a right-Cauchy sequence.

Since $(X, d)$ is a complete quasi metric space, then $\left(x_{n}\right)$ converges to some element $u \in X$. Therefore any subsequence of $\left(x_{n}\right)$ also converges to $u$. Thus the subsequences $\left(x_{2 n}\right)$ and $\left(x_{2 n+1}\right)$ also converge to $u$. Since $\left(x_{2 n}\right)$ is a sequence in $A, A$ is a closed subset of $X$ and $\lim _{n \rightarrow \infty} x_{2 n}=u$, then $u \in A$. Also, since $\left(x_{2 n+1}\right)$ is a sequence in $B, B$ is a closed subset of $X$ and $\lim _{n \rightarrow \infty} x_{2 n+1}=u$, then $u \in B$.

By using the continuity of $f$, we get

$$
\lim _{n \rightarrow \infty} d\left(x_{n}, f u\right)=\lim _{n \rightarrow \infty} d\left(f x_{n-1}, f u\right)=0 \text { and } \lim _{n \rightarrow \infty} d\left(f u, x_{n}\right)=\lim _{n \rightarrow \infty} d\left(f u, f x_{n-1}\right)=0 .
$$

Hence

$$
\lim _{n \rightarrow \infty} d\left(f u, x_{n}\right)=\lim _{n \rightarrow \infty} d\left(x_{n}, f u\right)=0 .
$$

Thus $\left(x_{n}\right)$ converges to $f u$. By uniqueness of the limit, we have $f u=u$. So $u$ is a fixed point of $f$ in $A \cap B$.

Now, since $u \preceq u$, then from $(2.2)$, we get

$$
\phi d(g u, u)=\phi d(g u, f u)
$$




$$
\begin{gathered}
\leq \quad \phi \max \{d(u, u), d(g u, u), d(f u, u)\} \\
-\psi \max \{d(u, u), d(g u, u), d(f u, u)\} .
\end{gathered}
$$

Thus $\phi d(g u, u) \leq \phi d(g u, u)-\psi d(g u, u)$. Hence $\psi d(g u, u)=0$, and so $d(g u, u)=0$. Therefore $g u=u$. Hence $u$ is a common fixed point for $f$ and $g$ in $A \cap B$.

Remark 2.5. The previous theorem is still correct if we choose the function $\psi:[0, \infty) \rightarrow[0, \infty)$ just as a continuous function.

Corollary 2.6. Let $(X, \preceq)$ be a partially ordered set and suppose that $(X, d)$ is a complete quasimetric space. Let $A, B$ be two nonempty closed subsets of $X$ with respect to the topology induced by $d$ with $X=A \cup B$ and $A \cap B \neq \phi$. Let $f: A \cup B \rightarrow A \cup B$ such that $f x \preceq f^{2} x$ for all $x \in X$ with $f(A) \subseteq B, f(B) \subseteq A$. Let $\phi, \psi$ be altering distance functions. Also suppose that

$$
\phi d(f x, f y) \leq \phi \max \{d(x, y), d(f x, x), d(f y, y)\}-\psi \max \{d(x, y), d(f x, x), d(f y, y)\}
$$

for all comparative $x, y \in X$ with $x \in A, y \in B$ or $x \in B, y \in$ A. Also,

1. suppose that there exists $x_{0} \in A$ such that $x_{0} \preceq f x_{0}$,

2. if $f$ or $g$ is continuous.

Then $f$ and $g$ have a common fixed point in $A \cap B$.

Proof. It follows from Theorem 2.4 by taking $g=f$.

Corollary 2.7. Let $(X, \preceq)$ be a partially ordered set and suppose that $(X, d)$ is a complete quasimetric space. Let $f, g: X \rightarrow X$ such that the pair $f$ and $g$ are weakly increasing mappings. Let $\phi, \psi$ be an altering distance functions. Moreover, suppose that

$$
\phi d(f x, g y) \leq \phi \max \{d(x, y), d(f x, x), d(g y, y)\}-\psi \max \{d(x, y), d(f x, x), d(g y, y)\}
$$

for all comparative $x, y \in X$, and

$$
\phi d(g x, f y) \leq \phi \max \{d(x, y), d(g x, x), d(f y, y)\}-\psi \max \{d(x, y), d(g x, x), d(f y, y)\}
$$

for all comparative $x, y \in X$. Also,

1. suppose that there exists $x_{0} \in A$ such that $x_{0} \preceq f x_{0}$,

2. if $f$ or $g$ is continuous.

Then $f$ and $g$ have a common fixed point in $A \cap B$.

Proof. It follows from Theorem 2.4 by taking $A=B=X$.

By replacing $g$ by $f$ and taking $A=B=X$ in Theorem 2.4 we get the following result.

Corollary 2.8. Let $(X, \preceq)$ be a partially ordered set and suppose that $(X, d)$ is a complete quasimetric space. Let $f: X \rightarrow X$ such that $f x \preceq f^{2} x$. Let $\phi, \psi$ be an altering distance functions. Moreover, suppose that

$$
\phi d(f x, f y) \leq \phi \max \{d(x, y), d(f x, x), d(f y, y)\}-\psi \max \{d(x, y), d(f x, x), d(f y, y)\}
$$

for all comparative $x, y \in X$. Also, 
1. suppose that there exists $x_{0} \in A$ such that $x_{0} \preceq f x_{0}$,

2. if $f$ or $g$ is continuous.

Then $f$ and $g$ have a common fixed point in $A \cap B$.

If we define $\phi:[0, \infty) \rightarrow[0, \infty)$ by $\phi(t)=t$ and $\psi:[0, \infty) \rightarrow[0, \infty)$ by $\psi(t)=(1-k) t, k \in[0,1)$, then we get the following result.

Theorem 2.9. Let $(X, \preceq)$ be a partially ordered set and suppose that $(X, d)$ is a complete quasimetric space. Let $A, B$ be two nonempty closed subsets of $X$ with respect to the topology induced by $d$ with $X=A \cup B$ and $A \cap B \neq \phi$. Let $f, g: A \cup B \rightarrow A \cup B$ such that the pair $(f, g)$ is (A,B)-weakly increasing with $f(A) \subseteq B, g(B) \subseteq A$. Suppose that

$$
d(f x, g y) \leq k \max \{d(x, y), d(f x, x), d(g y, y)\}
$$

for all comparative $x, y \in X$ with $x \in A, y \in B$, and

$$
d(g x, f y) \leq k \max \{d(x, y), d(g x, x), d(f y, y)\}
$$

for all comparative $x, y \in X$ with $x \in B, y \in A$. Also,

1. suppose that there exists $x_{0} \in A$ such that $x_{0} \preceq f x_{0}$,

2. if $f$ or $g$ is continuous.

Then $f$ and $g$ have a common fixed point in $A \cap B$.

Corollary 2.10. Let $(X, \preceq)$ be a partially ordered set and suppose that $(X, d)$ is a complete quasimetric space. Let $A, B$ be two nonempty closed subsets of $X$ with respect to the topology induced by $d$ with $X=A \cup B$ and $A \cap B \neq \phi$. Let $f: A \cup B \rightarrow A \cup B$ such that $f x \preceq f^{2} x$ forall $x \in X$ with $f(A) \subseteq B, f(B) \subseteq A$. Suppose that

$$
d(f x, f y) \leq k \max \{d(x, y), d(f x, x), d(f y, y)\}
$$

for all comparative $x, y \in X$ with $x \in A, y \in B$ or $x \in B, y \in$ A. Also,

1. suppose that there exists $x_{0} \in A$ such that $x_{0} \preceq f x_{0}$,

2. if $f$ or $g$ is continuous.

Then $f$ and $g$ have a common fixed point in $A \cap B$.

Proof. The proof follows from Theorem 2.9 by taking $g=f$.

Corollary 2.11. Let $(X, \preceq)$ be a partially ordered set and suppose that $(X, d)$ is a complete quasimetric space. Let $f, g: X \rightarrow X$ such that the pair $f$ and $g$ are weakly increasing. Suppose that

$$
d(f x, g y) \leq k \max \{d(x, y), d(f x, x), d(g y, y)\}
$$

for all comparative $x, y \in X$, and

$$
d(g x, f y) \leq k \max \{d(x, y), d(g x, x), d(f y, y)\}
$$

for all comparative $x, y \in X$. Also,

1. suppose that there exists $x_{0} \in A$ such that $x_{0} \preceq f x_{0}$, 
2. if $f$ or $g$ is continuous.

Then $f$ and $g$ have a common fixed point in $A \cap B$.

Proof. It follows from Theorem 2.9 by taking $A=B=X$

If we take $g=f$ and $A=B=X$ in Theorem 2.9, then we get the following result.

Corollary 2.12. Let $(X, \preceq)$ be a partially ordered set and suppose that $(X, d)$ is a complete quasimetric space. Let $f: X \rightarrow X$ such that $f x \preceq f^{2} x \forall x \in X$. Suppose that

$$
d(f x, f y) \leq k \max \{d(x, y), d(f x, x), d(f y, y)\}
$$

for all comparative $x, y \in X$, and

1. suppose that there exists $x_{0} \in A$ such that $x_{0} \preceq f x_{0}$,

2. if $f$ or $g$ is continuous.

Then $f$ and $g$ have a common fixed point in $A \cap B$.

\section{Common fixed point theorems in G-metric spaces}

Theorem 3.1. Let $(X, \preceq)$ be a partially ordered set and suppose that there exists a $G$-metric on $X$ such that $(X, G)$ is a complete $G$-metric space. Let $A, B$ be two nonempty closed subsets of $X$ with respect to the topology induced by $G$ with $X=A \cup B$. Let $f, g: A \cup B \rightarrow A \cup B$ be two mappings such that the pair $(f, g)$ is $(A, B)$-weakly increasing with $f(A) \subseteq B, g(B) \subseteq A$. Let $\phi$ and $\psi$ be an altering distance functions. Moreover, suppose that

$$
\begin{aligned}
\phi G(f x, g y, g y) \leq & \phi \max \{G(x, y, y), G(f x, x, x), G(g y, y, y)\} \\
& -\psi \max \{G(x, y, y), G(f x, x, x), G(g y, y, y)\}
\end{aligned}
$$

for all comparative $x, y \in X$ with $x \in A, y \in B$, and

$$
\begin{aligned}
\phi G(g x, f y, f y) \leq & \phi \max \{G(x, y, y), G(g x, x, x), G(f y, y, y)\} \\
& -\psi \max \{G(x, y, y), G(g x, x, x), G(f y, y, y)\}
\end{aligned}
$$

for all comparative $x, y \in X$ with $x \in B, y \in A$. Also

1. suppose that there exists $x_{0} \in A$ such that $x_{0} \preceq f x_{0}$,

2. if $f$ or $g$ is continuous.

Then $f$ and $g$ have a common fixed point in $A \cap B$.

Proof. Let $d: X \times X \rightarrow[0, \infty)$ defined by $d(x, y)=G(x, y, y)$ for all comparative $x, y \in X$ with $x \in A, y \in B$ and $d(y, x)=G(y, x, x)$ for all comparative $x, y \in X$ with $x \in A, y \in B$. Then by Theorem 1.14, $(X, d)$ is a quasi metric space. From the contractive conditions we have

$$
\phi d(f x, g y) \leq \phi \max \{d(x, y), d(f x, x), d(g y, y)\}-\psi \max \{d(x, y), d(f x, x), d(g y, y)\}
$$

for all comparative $x, y \in X$ with $x \in A, y \in B$, and

$$
\phi d(g x, f y) \leq \phi \max \{d(x, y), d(g x, x), d(f y, y)\}-\psi \max \{d(x, y), d(g x, x), d(f y, y)\}
$$

for all comparative $x, y \in X$ with $x \in B, y \in A$. By Theorem 2.4, $f$ and $g$ have a common fixed point in $A \cap B$. 
Theorem 3.2. Let $(X, \preceq)$ be a partially ordered set and suppose that there exists a $G$-metric on $X$ such that $(X, G)$ is a complete $G$-metric space. Let $A$ and $B$ be two nonempty closed subsets of $X$ with respect to the topology induced by $G$ with $X=A \cup B$. Let $f, g: A \cup B \rightarrow A \cup B$ be two mappings such that the pair $(f, g)$ is $(A, B)$-weakly increasing with $f(A) \subseteq B$ and $g(B) \subseteq A$. Suppose that there exists $r \in[0,1)$ such that

$$
G(f x, g y, g y) \leq k \max \{G(x, y, y), G(f x, x, x), G(g y, y, y)\}
$$

for all comparative $x, y \in X$ with $x \in A, y \in B$, and

$$
G(g x, f y, f y) \leq k \max \{G(x, y, y), G(g x, x, x), G(f y, y, y)\}
$$

for all comparative $x, y \in X$ with $x \in B, y \in A$. Also,

1. suppose that there exists $x_{0} \in A$ such that $x_{0} \preceq f x_{0}$,

2. if $f$ or $g$ is continuous.

Then $f$ and $g$ have a common fixed point in $A \cap B$.

Proof. As in the proof of Theorem 3.1, we consider the function $d: X \times X \rightarrow[0, \infty)$ such that $d(x, y)=G(x, y, y)$ for all comparative $x, y \in X$ with $x \in A, y \in B$ and $d(y, x)=G(y, x, x)$ for all comparative $x, y \in X$ with $x \in A, y \in B$. Then by Theorem 1.14, $(X, d)$ is a quasi metric space. From the contractive conditions we have

$$
d(f x, g y) \leq k \max \{d(x, y), d(f x, x), d(g y, y)\}
$$

for all comparative $x, y \in X$ with $x \in A, y \in B$, and

$$
d(g x, f y) \leq k \max \{d(x, y), d(g x, x), d(f y, y)\}
$$

for all comparative $x, y \in X$ with $x \in B, y \in A$. By Theorem 2.9, $f$ and $g$ have a common fixed point in $A \cap B$.

Remark 3.3. We can prove Theorem 3.2 from Theorem 3.1 by choosing $\phi t=t$ and $\psi t=(1-k) t$, where $0 \leq k<1$.

Next, we introduce an example to support our result.

Example 3.4. Let $X=\{0,1,2,3, \cdots\}$ and define a relation $\preceq$ on $X$ by $a, b \in X, a \preceq b$ iff $a-b \geq 0$ and let $A$ and $B$ be two subsets of $\mathrm{X}$ such that $A=\{0,2,4,6, \cdots\}, B=\{0,1,3,5, \cdots\}$.

Define $d: X \times X \rightarrow[0, \infty)$ by $d(x, y)= \begin{cases}0, & x=y \\ x+2 y, & x \neq y\end{cases}$

Let $f, g: A \cup B \rightarrow A \cup B$ be defined by $f x=\left\{\begin{array}{ll}0, & x=0,1,2 ; \\ x-3, & x \geq 3 .\end{array} \quad g x= \begin{cases}0, & x=0,1 \text {; } \\ x-1, & x \geq 2 .\end{cases}\right.$ Also, define $\phi, \psi:[0, \infty) \rightarrow[0, \infty)$ by $\phi t=t^{2}, \psi t=t$. Then

(1) $(X, d, \preceq)$ is a partially ordered complete quasi metric space;

(2) $A$ and $B$ are closed subsets of $X$ with respect to the topology induced by $d$;

(3) the pare $(f, g)$ is $(A, B)$-weakly increasing with $f(A) \subseteq B, g(B) \subseteq A$;

(4) $\phi$ and $\psi$ are altering distance functions;

(5) there is $x_{0} \in X$ such that $x_{0} \preceq f x_{0}$; 
(6)

$$
\phi d(f x, g y) \leq \phi \max \{d(x, y), d(f x, x), d(g y, y)\}-\psi \max \{d(x, y), d(f x, x), d(g y, y)\}
$$

for all comparative $x, y \in X$ with $x \in A, y \in B$, and

$$
\phi d(g x, f y) \leq \phi \max \{d(x, y), d(g x, x), d(f y, y)\}-\psi \max \{d(x, y), d(g x, x), d(f y, y)\}
$$

for all comparative $x, y \in X$ with $x \in B, y \in A$.

Proof. The proofs of (1), (2), (3), (4), and (5) are clear. We show (6).

Let $x \in A, y \in B$. Then we have the following cases:

Case (I): If $x \in\{0,1,2\}$ and $y \in\{0,1\}$, then $f x=0$ and $g y=0$. Hence the left hand side of (3.1) is equal to 0 and so 3.1 is satisfied.

Case (II): If $x \geq 3$ and $y \geq 2$, then

Subcase (1): If $x-3=y-1$, then $\phi d(f x, g y)=[d(x-3, y-1)]^{2}=[0]^{2}=0$ and so (3.1) is satisfied.

Subcase (2): If $x-3 \neq y-1$, then

$$
\phi d(f x, g y)=[d(x-3, y-1)]^{2}=[x+2 y-5]^{2}=x^{2}+4 y^{2}+25+4 x y-10 x-20 y .
$$

On the other hand

$$
\begin{aligned}
{[\max } & \{d(x, y), d(f x, x), d(g y, y)\}]^{2}-\max \{d(x, y), d(f x, x), d(g y, y)\} \\
& =[\max \{d(x, y), d(x-3, x), d(y-1, y)\}]^{2}-\max \{d(x, y), d(x-3, x), d(y-1, y)\} \\
& =[\max \{x+2 y, 2 x-3,2 y-1\}]^{2}-\max \{x+2 y, 2 x-3,2 y-1\} .
\end{aligned}
$$

If $\max \{x+2 y, 2 x-3,2 y-1\}=x+2 y$, then the right hand side is $x^{2}+4 y^{2}+4 x y-x-2 y$. Assume to the contrary that $x^{2}+4 y^{2}+25+4 x y-10 x-20 y>x^{2}+4 y^{2}+4 x y-x-2 y$. Then we have $9 x+18 y<25$ a contradiction since $x \geq 3$ and $y \geq 2$. Thus we have $x^{2}+4 y^{2}+25+4 x y-10 x-20 y \leq$ $x^{2}+4 y^{2}+4 x y-x-2 y$. If $\max \{x+2 y, 2 x-3,2 y-1\}=2 x-3$ or $2 y-1$, then the result is clear since if $a, b \in \mathbb{N}$ with $a<b$, then $a^{2}-a<b^{2}-b$. Thus (3.1) is satisfied. In a similar manner we can show that $(3.2)$ is satisfied. Hence all hypothesis of Theorem 3.1 hold true. Therefore $f$ and $g$ have a common fixed point in $A \cap B$. In this example the common fixed point of $f$ and $g$ in $A \cap B$ is 0 .

\section{Acknowledgment}

The authors would like to acknowledge the grant: UKM Grant DIP-2014-034 and Ministry of Education, Malaysia grant FRGS/1/2014/ST06/UKM/01/1 for financial support.

\section{References}

[1] R. P. Agarwal, M. A. El-Gebeily, D. O'Regan, Generalized contractions in partially ordered metric spaces, Appl. Anal., 87 (2008), 109-116. 1, 1.1.

[2] R. P. Agarwal. E. Karapinar, A. F. Roldán-López-de-Hierro, Last remarks on G-metric spaces and related fixed point theorems, Rev. R. Acad. Cienc. Exactas Fs. Nat. Ser. A Math. RACSAM, 110 (2016), 433-456. 1

[3] I. Alyun, H. Simsek, Some fixed point theorems on orderd metric spaces and applications, Fixed Point Theory Appl., 2010 (2010), 17 pages. 2.1 
[4] S. Banach, Sur les opérations dans les ensembles abstraits et leur application aux équations intégrales, Fund. Math., 3 (1922), 133-181. 1

[5] M. Jleli, B. Samet, Remarks on G-metric spaces and fixed point theorems, Fixed Point Theory Appl., 2012 (2012), 7 pages. 1, 1.3, 1.4, 1.5, 1.6, 1.7, 1.14

[6] M. S. Khan, M. Swaleh, S. Sessa, Fixed point theorems by altering distances between the points, Bull. Austral. Math. Soc., 30 (1984), 1-9. 2.3

[7] N. V. Luong, N. X. Thuan, Coupled fixed point theorems in partially ordered G-metric spaces, Math. Comput. Modelling, 55 (2012), 1601-1609. 1]

[8] Z. Mustafa, Common fixed points of weakly compatible mappings in G-metric spaces, Appl. Math. Sci. (Ruse), 6 (2012), 4589-4600.

[9] Z. Mustafa, Some new common fixed point theorems under strict contractive conditions in G-metric spaces, J. Appl. Math., 2012 (2012), 21 pages.

[10] Z. Mustafa, M. Khandaqji, W. Shatanawi, Fixed point results on complete G-metric spaces, Studia Sci. Math. Hungar., 48 (2011), 304-319.

[11] Z. Mustafa, H. Obiedat, A fixed point theorem of Reich in G-metric spaces, Cubo, 12 (2010), 83-93.

[12] Z. Mustafa, H. Obiedat, F. Awawdeh, Some fixed point theorem for mapping on complete G-metric spaces, Fixed Point Theory Appl., 2008 (2008), 12 pages.

[13] Z. Mustafa, W. Shatanawi, M. Bataineh, Existence of fixed point results in G-metric spaces, Int. J. Math. Math. Sci., 2009 (2009), 10 pages.

[14] Z. Mustafa, B. Sims, A new approach to generalized metric spaces, J. Nonlinear Convex Anal., 7 (2006), 289-297. 1, 1, 1.8, 1.9, 1.10, 1.11, $1.12,1.13$

[15] Z. Mustafa, B. Sims, Fixed point theorems for contractive mappings in complete G-metric spaces, Fixed Point Theory Appl., 2009 (2009), 10 pages.

[16] B. Samet, C. Vetro, F. Vetro, Remarks on G-metric spaces, Int. J. Anal., 2013 (2013), 6 pages. 1

[17] W. Shatanawi, M. Postolache, Some fixed-point results for a G-weak contraction in G-metric spaces, Abstr. Appl. Anal., 2012 (2012), 19 pages.

[18] W. Shatanawi, M. Postolache, Common fixed point results for mappings under nonlinear contraction of cyclic form in ordered metric spaces, Fixed Point Theory Appl., 2013 (2013), 13 pages. 1, 2.2

[19] W. A. Wilson, On quasi-metric spaces, Amer. J. Math., 53 (1931), 675-684. 1 ] 